PASCUAL-SALCEDO Grant/research support from: Pfizer, Speakers bureau: Pfizer, Abbvie, Takeda, Eulalia Rodríguez-Martín: None declared, Chamaida Plasencia Speakers bureau: Pfizer, MSD

DOI: 10.1136/annrheumdis-2019-eular.3244

\section{FRI0096 TNF CONCENTRATIONS DURING TREATMENT OF INFLAMMATORY DISEASES WITH GOLIMUMAB}

Femke Hooijberg ${ }^{1}$, Lea C. Berkhout ${ }^{2,3}$, Merel J. L'ami ${ }^{1}$, Sadaf Atiqi ${ }^{1}$, Michael Nurmohamed ${ }^{1}$, Annick de Vries ${ }^{4}$, Charlotte L. Krieckaert ${ }^{5}$, Theo Rispens ${ }^{2,3}$, Gert-Jan Wolbink ${ }^{1,2,3}$. ${ }^{1}$ Amsterdam Rheumatology and immunology Center, location Reade, Amsterdam, Netherlands; ${ }^{2}$ Department of Immunopathology, Sanquin Research, Amsterdam, Netherlands; ${ }^{3}$ Landsteiner Laboratory, Academic Medical Centre, University of Amsterdam, Amsterdam, Netherlands; ${ }^{4}$ Biologics Lab, Sanquin Diagnostic Services, Amsterdam, Netherlands; ${ }^{5}$ Amsterdam Rheumatology and immunology Center, location VUmc, Amsterdam, Netherlands

Background: Golimumab is a monoclonal antibody that binds TNF, counteracting its pro-inflammatory effects. Berkhout et al. (2019) described the development of a novel drug-tolerant assay to quantify TNF during TNFinhibitor treatment. Using this assay, they analyzed the dynamics of TNF during treatment of rheumatoid arthritis (RA) patients with adalimumab and found that TNF levels shortly after treatment initiation predict nonresponse (1). Our study is the first to measure TNF levels during golimumab treatment, using the same drug-tolerant assay.

Objectives: To describe the dynamics of TNF in patients with RA, ankylosing spondylitis (AS) or psoriatic arthritis (PsA) during golimumab treatment.

Methods: Consecutive patients with RA, AS, or PsA starting golimumab treatment were included in this prospective observational cohort study, named the Reade Rheumatology Registry. Serum samples drawn during the study visits were analyzed for drug levels, anti-drug antibody (ADA) formation, and TNF concentrations using a regular enzyme-linked immunosorbent assay (ELISA), a radioimmunoassay, and a drug-tolerant competition ELISA, respectively. Regression analyses were used to analyze the data. Missing data was imputed using last observation carried forward.

Results: In total, 304 serum samples from 69 patients were included in this study. The median follow-up period was 52 (interquartile range (IQR) 16-130) weeks. Median TNF concentration at baseline was 4 (IQR 3-105) $\mathrm{pg} / \mathrm{ml}$ and this increased to $68(37-127) \mathrm{pg} / \mathrm{ml}$ four weeks after treatment initiation. During follow-up, TNF concentrations remained stable for the majority of patients (Figure 1). TNF levels at baseline were already high $(\geq 30 \mathrm{pg} / \mathrm{ml})$ in $25 \%$ of the patients. These patients used a previous TNF-inhibitor with a median of 19 (IQR 11-32) days before start of golimumab. In contrast, patients with low baseline TNF concentrations were TNF-inhibitor naive, used a TNF-inhibitor longer before the start of golimumab treatment (median 489 (IQR 56-816) days), or used a biologic with a different target. A trend was found for the association between drug level and TNF concentration during follow-up; patients with low TNF concentrations $(<30 \mathrm{pg} / \mathrm{ml})$ had median drug levels of 0.32 (IQR $0.03-$ 1.00) $\mu \mathrm{g} / \mathrm{ml}$, while patients with high TNF concentrations $(\geq 30 \mathrm{pg} / \mathrm{ml}$ ) reached a median drug level of $1.45(0.80-2.47) \mu \mathrm{g} / \mathrm{ml}$ (odds ratio (OR) 1.51, Cl: $0.97-2.37, p=0.071$ ). No association was found between TNF concentrations at week 4 and low disease activity at week 52, defined as DAS28 $\leq 3.2$ or ASDAS $<2.1$ (OR $1.00, \mathrm{Cl}$ : 1.00-1.00, $\mathrm{p}=309$ ). Four patients $(6 \%)$ developed detectable ADAs.

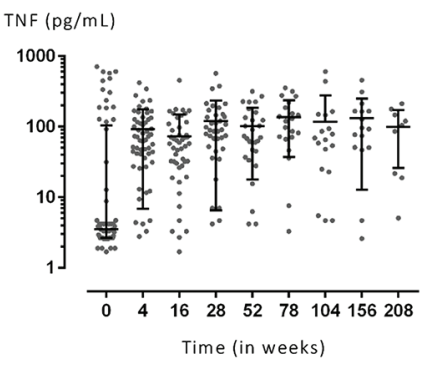

Figure 1

Conclusion: Golimumab therapy was found to induce an increase in TNF concentrations, independent of disease activity. This is in line with what has been found for adalimumab treatment. However, TNF concentrations measured in this study were at least twice as low as TNF concentrations during adalimumab treatment (1). The high baseline TNF concentrations in $25 \%$ of the patients were also surprising, as it indicates that patients switch to golimumab while their TNF is still in complex with a previous TNF-inhibitor.

\section{REFERENCE:}

[1] L.C. Berkhout, M.J. I'Ami, J. Ruwaard, M.H. Hart, P.O.-d. Heer, K. Bloem, M.T. Nurmohamed, R.F. Vollenhoven, M. Boers, D.F. Alvarez, C.H. Smith, G.J. Wolbink, T. Rispens, Dynamics of circulating TNF during adalimumab treatment using a drug-tolerant TNF assay. Sci. Transl. Med. 11, eaat3356 (2019).

Disclosure of Interests: Femke Hooijberg: None declared, Lea C. Berkhout: None declared, Merel J. I'Ami: None declared, Sadaf Atiqi: None declared, Michael Nurmohamed Grant/research support from: AbbVie, Bristol-Myers Squibb, Celgene, Eli Lilly, Janssen, Menarini, MSD, Mundipharma, Pfizer, Roche, Sanofi and UCB, Consultant for: AbbVie, BristolMyers Squibb, Celgene, Eli Lilly, Janssen, Menarini, MSD, Mundipharma Pfizer, Roche, Sanofi and UCB, Speakers bureau: AbbVie, Bristol-Myers Squibb, Celgene, Eli Lilly, Janssen, Menarini, MSD, Mundipharma, Pfizer, Roche, Sanofi and UCB, Annick de Vries: None declared, Charlotte L. Krieckaert: None declared, Theo Rispens Grant/research support from: Genmab, Speakers bureau: Pfizer, Abbvie, Regeneron, Gert-Jan Wolbink Grant/research support from: Pfizer (paid to the institution), Speakers bureau: Pfizer, UCB, AbbVie, Biogen, BMS

DOI: 10.1136/annrheumdis-2019-eular.1908

\section{FRI0097 RA PATIENTS' PERSPECTIVES ON BIOLOGICAL DMARD-INDUCED ADVERSE DRUG REACTIONS AND THEIR BURDEN}

Jette van Lint ${ }^{1}$, Naomi Jessurun ${ }^{1}$, Sander W. Tas ${ }^{2}$, Harald Vonkeman ${ }^{3}$, Bart van den Bemt ${ }^{4,5}$, Astrid van Tubergen ${ }^{6}$, Michael Nurmohamed ${ }^{7,8}$, Eugène van Puijenbroek ${ }^{1,9} .{ }^{1} N$ Netherlands Pharmacovigilance Centre Lareb, 'sHertogenbosch, Netherlands, ${ }^{2}$ Amsterdam University Medical Centers, location AMC, Rheumatology and Clinical Immunology, Amsterdam, Netherlands; ${ }^{3}$ Medisch Spectrum Twente, Rheumatology and Clinical Immunology, Enschede, Netherlands; ${ }^{4}$ Sint Maartenskliniek, Nijmegen, Netherlands; ${ }^{5}$ Radboudumc, Nijmegen, Netherlands; ${ }^{6}$ Maastricht UMC+, Rheumatology, Maastricht, Netherlands; ${ }^{7}$ Amsterdam Rheumatology and Immunology Center Reade, Amsterdam, Netherlands; ${ }^{8}$ Amsterdam University Medical Centers, location VUMC, Rheumatology and Clinical Immunology, Amsterdam, Netherlands; ${ }^{9}$ University of Groningen, Research Institute of Pharmacy, Groningen, Netherlands

Background: Numerous biological DMARDs (bDMARDs) are used in RA treatment, however detailed knowledge of patients' perceptions on drug use and the impact of adverse drug reactions (ADRs) is sparse.

Objectives: To gain insight into bDMARD-induced ADRs and their burden from the RA patients' perspective.

Methods: The Dutch Biologic Monitor is an ADR-patient web-based questionnaire used for a prospective, multicentre, event monitoring cohort study including patients using a bDMARD for an immune-mediated inflammatory disease between January 1, 2017 and December 31, 2018 Patients were asked to complete questionnaires bi-monthly about used bDMARDs, indication and bDMARD-induced ADRs. ADRs were coded according to MedDRA terminology and their impact was measured on a 5-point scale, ranging from 1 (no burden) to 5 (very high burden). Per patient, every recurrent unique ADR was included as one ADR. ADRs regarding infections (INF), skin (SK), gastrointestinal (Gl) and injection site (INJ) were clustered and analysed for the reported prevalence and burden. Fatigue and headache were separately analysed for prevalence and burden. The prevalence of clustered ADRs between the various bDMARDs was compared using a $\chi^{2}$ test and the average burden was compared using a Mann-Whitney $U$ test.

Results: In the Dutch Biologic Monitor 583 consecutive (44.8\%) RA patients were included $(71.2 \%$ female, average age 59 years, SD \pm 12.4 using the originator or a biosimilar of etanercept (ETN, 265), adalimumab (ADA, 196), tocilizumab (41), abatacept (35), certolizumab pegol (23), rituximab (19), infliximab (18), golimumab (15), sarilumab (2), secukinumab (1), anakinra (1). Almost half of the patients $(276 ; 47.3 \%)$ reported at least one bDMARD-induced ADR with a total of 703 ADRs in 2,559 completed questionnaires. Patients reported 129 INJ reactions (129 ADRs/ 583 pts, prevalence of $22.1 \%$, reported by 86 pts) with an average 